\title{
Is amygdala size correlated with stress?
}

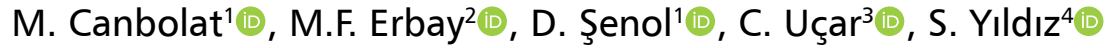 \\ ${ }^{1}$ Department of Anatomy, Inönü University, Malatya, Turkey \\ 2Department of Radiology, Inönü University, Malatya, Turkey \\ ${ }^{3}$ Department of Physiology, Adıyaman University, Adıyaman, Turkey \\ ${ }^{4}$ Department of Physiology, Inönü University, Malatya, Turkey
}

[Received: 18 June 2020; Accepted: 10 August 2020; Early publication date: 14 August 2020]

\begin{abstract}
Background: One of the important mechanisms that regulate the stress response of the body is hypothalamic pituitary adrenal axis. One of the structures activating this axis is amygdala. We have seen people around who react calmer and cooler to very stressful situations. Are people with smaller amygdala really calmer? Or, can we say that the bigger the amygdala, which is the trigger of the body's response to stress, the more a person panics? Aim of the study is to compare the saliva cortisol levels and amygdala volume.

Materials and methods: Study conducted with 63 male students. Magnetic resonance images of students were taken before their final exam to calculate amygdala volumes. Saliva samples of all students were taken two times to detect cortisol levels in saliva. First one was 20 days before the final exam and second one was on the exam day. We assumed that the students were stressful on exam day. Results and Conclusions: No statistically significant correlation was found between saliva cortisol levels and amygdala volume in the study. (Folia Morphol 2021; 80, 3: 514-519)
\end{abstract}

Key words: amygdala volume, saliva cortisol, stress, magnetic resonance images

\section{INTRODUCTION}

One of the most basic problems of today's human beings is stress. Stress, which is defined as the sensual tension $[2,35]$ that occurs as a result of the deterioration of physiological and spiritual well-being of the organism due to environmental factors, has negative influences on individuals' self-respect and productivity. Since stress plays a significant role in the occurrence of many diseases, one of the most important goals of health professionals is to eliminate stress and if this is not possible, to manage stress [22]. The response given by organism to stress in acute period is a useful tool that activates the resources of the organism to protect it against a dangerous situation [26]. Low levels of stress can even be instructional for the organism and can cause the organism to find new solutions to a problem [27]. What causes distress is the persistence and chronicity of stress. While acute and low intensity stress is useful for a person, chronic stress damages cognitive functions and neurological structures such as the hippocampus [10,20].

One of the important mechanisms that regulate the stress response of the body in case of a stressful situation is hypothalamic pituitary adrenal axis (HPA axis) [29]. The function of HPA axis is sensing the external stimulus, assessing the stimulus and creating

Address for correspondence: Dr. M. Canbolat, Department of Anatomy, Inönü University Faculty of Medicine, 44280 Malatya, Turkey, tel: +090 5056682547, fax: +090 4223410036, e-mail: mustafa.canbolat@inonu.edu.tr

This article is available in open access under Creative Common Attribution-Non-Commercial-No Derivatives 4.0 International (CC BY-NC-ND 4.0) license, allowing to download articles and share them with others as long as they credit the authors and the publisher, but without permission to change them in any way or use them commercially. 
a fight-or-run response, completing the fight in the shortest time possible and bringing the organism back to its normal state. One of the structures activating this axis is amygdala. Amygdala is an almond-shaped subcortical structure in the temporal region of our brain. Amygdala, which is part of the limbic system, assesses the sensory direction of the external stimulus [31]. In addition, it realizes the activation or inhibition of responses like fear and anxiety [18]. It protects the organism from danger and enables its survival. It plays an active role in triggering emotions against external stimuli [32]. Amygdala assesses every situation and every object and makes this assessment roughly with questions such as "is it something that I like?", "can this harm me?", and if it is accepted as dangerous as a result of the assessment, amygdala starts the crisis situation. Long before an external sensory stimulus reaches the cortex after being filtered from the thalamus; it reaches the amygdala in a much faster way. By the time the sense has reached the cortex and has been assessed, amygdala has already started the process $[16,21]$. When amygdala that has sensed the danger is activated, it sends a signal to hypothalamus and corticotrophin releasing factor (CRF) is released from the hypothalamus. CRF causes the pituitary to release adrenocorticotropic hormone (ACTH) and ACTH enables cortisol release from adrenal gland [11]. Cortisol regulates the physiological response of the organism against stress.

The thought that there might be an association between amygdala size and responses given under stress does not seem to be unreasonable because stimulating the amygdala causes fear and anxiety. We have seen people around who react calmer and cooler to very stressful situations. Are people with smaller amygdala really calmer? Or, can we say that the bigger the amygdala, which is the trigger of the body's response to stress, the more a person panics? In our study, our aim was to compare the saliva cortisol levels of healthy university students exposed to the same stressor and their amygdala size in order to find an answer to this question.

\section{MATERIALS AND METHODS}

Ethical approval for our study was obtained from the Malatya Clinical Researches Ethical Board (No. 2018/111). The participants in our study were informed about the study and they read and signed the informed consent form. We conducted our study with 63 male students from Inönü University, Faculty of Medicine who were not using cigarette, alcohol or drugs, who did not have any psychological disease, who did not have any seizures in childhood and later, who did not have any head trauma or surgical operation and who were right-handed. Median age of students were 21 (min: 19, max: 27) years.

We accepted 20 days before the final exam as relaxed period. Exam day is accepted as stressed period. We assumed that the students were stressed on exam day. To support this idea we used cortisol levels and State Trait Anxiety Inventory-I (STAI-I). The STAI-I was given to students during relaxed and stressed periods. Also saliva samples were taken during relaxed and stressed periods to detect cortisol levels in saliva. The STAI-I is one of the common scales to assess anxiety [4]. According to STAI-I, $\leq 36$ points means "no anxiety", 37-42 points means "mild anxiety", $\geq 42$ points means "high anxiety".

\section{Neuroimaging}

We got the magnetic resonance (MR) images of students a day before exam. 3T Siemens scanner (Skyra syngo MR E11 version, Germany) was used for neuroimaging. T1-weighted three-dimensional (3D) (MPRAGE) sequence was used in sagittal plane to get structural images and the following parameters were used: TE/TR: $2300 \mathrm{~ms} / 2.32 \mathrm{~s}$, flip angle: $8^{\circ}$, field of view: $240 \mathrm{~mm}^{2}$, slice thickness: $0.9 \mathrm{~mm}$. MR T1 data was downloaded from the scanner and by using different software, they were transferred and processed. MR images were kept in hdr and img formats. To do this, a personal computer on a 32-bit Dell PC, running Windows 10 operating system was used. Volume was calculated with mricloud (www.mricloud.org). Installation, configuration or training are not required to use the web based module. Through a web interface, mricloud volumetric analysis system functions remotely to provide a report including volumetric information from any submitted case.

\section{Analysis saliva cortisol}

Saliva samples of all students were taken before they entered the exam. Saliva samples were collected by using passive drool method [8]. Samples collected were kept in a laboratory freezer at $-20^{\circ} \mathrm{C}$. After thawing, the samples were centrifuged for $10 \mathrm{~min}$ at $4000 \mathrm{~g}$ and enzyme-linked immunosorbent assay (ELISA) analyses were conducted by using the supernatant. 
Table 1. Values of cortisol levels and State Trait Anxiety Inventory-I (STAI-I) scores of volunteers

\begin{tabular}{|c|c|c|c|c|c|}
\hline \multirow[t]{2}{*}{ Variables } & \multicolumn{2}{|c|}{ Relaxed } & \multicolumn{2}{|c|}{ Stressed } & \multirow[t]{2}{*}{$\mathbf{P}$} \\
\hline & Median & Minimum-Maximum & Median & Minimum-Maximum & \\
\hline Cortisol levels & 8.34 & $2.87-24.16$ & 15.45 & $2.99-122.78$ & 0.000 \\
\hline STAI-I score & 32 & $21-68$ & 58 & $24-71$ & 0.000 \\
\hline
\end{tabular}

Table 2. Correlation analysis of amygdala volumes with cortisol level differences and State Trait Anxiety Inventory-I (STAI-I) score differences between relaxed and stressed periods

\begin{tabular}{lccc}
\hline Variables & $\begin{array}{c}\text { Test } \\
\text { statistics }\end{array}$ & $\begin{array}{c}\text { Cortisol } \\
\text { difference }\end{array}$ & $\begin{array}{c}\text { STAI-I score } \\
\text { difference }\end{array}$ \\
\hline Right amygdala & $\mathrm{r}$ & -0.120 & 0.120 \\
volume & $\mathrm{p}$ & 0.350 & 0.351 \\
Left amygdala & $\mathrm{r}$ & -0.156 & 0.136 \\
volume & $\mathrm{p}$ & 0.222 & 0.287 \\
Total amygdala & $\mathrm{r}$ & -0.146 & 0.120 \\
volume & $\mathrm{p}$ & 0.253 & 0.347 \\
\hline
\end{tabular}

Table 3. Numbers of volunteers in relaxed and stressed periods according to State Trait Anxiety Inventory-I (STAI-I) scores

\begin{tabular}{lccc}
\hline Period & \multicolumn{3}{c}{ STAI-I scores } \\
\cline { 2 - 4 } & $\begin{array}{c}<\mathbf{3 6} \text { (no } \\
\text { anxiety) }\end{array}$ & $\begin{array}{c}\mathbf{3 7 - 4 2} \text { (mild } \\
\text { anxiety) }\end{array}$ & $\begin{array}{c}\mathbf{>} \mathbf{4 2} \text { (high } \\
\text { anxiety) }\end{array}$ \\
\hline Relaxed & $35(55.6 \%)$ & $19(30.1 \%)$ & $9(14.3 \%)$ \\
Stressed & $4(6.3 \%)$ & $6(9.6 \%)$ & $53(84.1 \%)$ \\
\hline
\end{tabular}

All samples were diluted at 1:5 and assayed in triplicate by using the assay buffer. Carbonate buffer, $\mathrm{pH}$ 9.6 was used to dilute cortisol-bovine serum albumin stock solution ( $1 \mathrm{mg} / \mathrm{mL}$ ) by ELISA procedure and this was added to 96 -well microtiter plate at $200 \mu \mathrm{L} /$ well. Later, the microtiter plate was incubated at $+4^{\circ} \mathrm{C}$ for a night and washed 5 times with washing buffer by using an 8-channel pipette. Some of the binding places that did not include coating antigen were blocked for 2 hours at $37^{\circ} \mathrm{C}$ with blocking buffer (200 $\mu \mathrm{L}$ ) /well). Following the process of washing, diluted first Ab (antiserum) (40 $\mu \mathrm{L} /$ well) and standard solutions or samples ( $40 \mu \mathrm{L} /$ well) were placed in duplicate and incubated at $37^{\circ} \mathrm{C}$ for $45 \mathrm{~min}$. After the washing process, biotinylated anti-rabbit antibody (100 $\mu \mathrm{L} /$ well) was added and the plate was incubated at $37^{\circ} \mathrm{C}$ for $30 \mathrm{~min}$. After washing for 5 times, Streptavidin peroxidase solution (100 $\mu \mathrm{L} /$ well) was added and the plate was incubated for $15 \mathrm{~min}$ at $+4^{\circ} \mathrm{C}$. Following another 5 times of washing, substrate solution
(150 $\mu \mathrm{L} /$ well) was added to the plate and incubated in dark for $10 \mathrm{~min}$. Following incubation, stop solution (50 $\mu \mathrm{L} /$ well) was also added and absorbance was measured at $450 \mathrm{~nm}$ by using the microplate reader. Inter-assay variation was found to be $7.8 \%$, while inter-assay coefficients of variation was $5.6 \%$.

\section{Statistical analysis}

Kolmogorov-Smirnov test was used to find out whether the data were normally distributed. The Wilcoxon paired-samples test was used to analyse the data which were not normally distributed. Spearman Rho correlation analysis was conducted on the data to find out how the cortisol level differed with amygdala volume and STAI-I scores. Minimum ( $\mathrm{min}$ ) and maximum ( $\max$ ) values of data which were not normally distributed were given with median. Mann-Whitney $U$ test was performed to compare the right and left amygdala volumes. $\mathrm{P}<0.05$ values were considered as statistically significant. IBM SPSS Statistics 22.0 for Windows program was used in statistical analyses.

\section{RESULTS}

The median value of saliva cortisol increased in stressed period. Also STAI-I score increased in stressed period. The Wilcoxon paired-samples test was conducted on data and statistically significant increase was found in relaxed and stressed period saliva cortisol and STAI-I scores (Table 1).

As a result of Spearman rho correlation analysis of amygdala volumes and difference in cortisol values between relaxed period and stressed period, it was found that there was no correlation. Also there was no correlation between amygdala volumes and STAI-I score differences of relaxed and stressed periods (Table 2 ).

For the STAI-I score, there is a decrease in numbers of "no anxiety" and "mild anxiety" volunteers in stressed period compared to relax period. But there is a high increase in stressed period for the numbers of "high anxiety" volunteers compared to relax period (Table 3). 
Table 4. Right, left and total amygdala volumes of volunteers

\begin{tabular}{lcc}
\hline Variables & Amygdala volume $\left[\mathrm{mm}^{3}\right]$ & $\mathbf{P}$ \\
\hline Right amygdala & $1974(1533-2356)$ & 0.000 \\
Left amygdala & $1781(1480-2063)$ & \\
Total amygdala & $3681(3055-4349)$ & \\
\hline
\end{tabular}

Median value of the right amygdala volume of our volunteers was found as 1974 (1533-2356) $\mathrm{mm}^{3}$, while the median value of their left amygdala volume was 1781 (1480-2063) $\mathrm{mm}^{3}$. Median value of total amygdala volume of our volunteers was found 3681 (3055-4349) $\mathrm{mm}^{3}$. Mann-Whitney $U$ test was conducted on the data to find out whether the difference was significant. We found a statistically significant difference between the volumes of right and left amygdala (Table 4).

\section{DISCUSSION AND CONCLUSIONS}

For a high efficiency from personnel working in occupations with high stress; mainly security staff, surgeons and athletes; individuals need to be able to cope with these stressful situations. Of course, it is important to show quantitatively at the beginning of personnel choice whether the individual has such an ability. We conducted this study with the thought that we could obtain a parameter in the detection of personnel to be employed in the aforementioned stressful fields if we could find an association between amygdala volume and cortisol level.

In our study, we received the saliva cortisol level as an indicator of stress. As mentioned above, the ultimate product of the HPA axis, which is activated as a result of stress, is cortisol that is released in the circulation [17]. Cortisol level in saliva reflects the serum cortisol level $[5,13]$. We chose to use saliva cortisol as an indicator of stress due to reasons such as its being non-invasive, not putting the samples in an extra stress and the ease of taking samples.

As a result of our study, we could not find an association between amygdala size and cortisol values we obtained from the samples as a quantitative parameter. In studies conducted, a big amygdala size has been associated with increased anxiety [1], being sensitive to negative experiences [6] and negative affectivity [9]. On the other hand, studies conducted in patients with unipolar depressive disorder have not shown a significant association between amygdala size and basal cortisol release [12, 25]. In another study conducted in patients with major depression, a positive correlation was found between amygdala size and average cortisol level [19]. In another study, a correlation was found between amygdala activities of university students exposed to the same stressor and the environment they lived in. While the bigger and more stressful place individuals lived in the more amygdala activity they had, subjects who lived in rural areas were found to have low amygdala activity [15]. Being exposed to the same stressor but responding differently can also be associated with amygdala size. In a study conducted with healthy female children, a positive correlation was found between fear and amygdala size [33]. In a study conducted on children who spent the first 2 years of their lives in an orphanage and who were exposed to abuse, amygdalae of these children were found to be big [30]. Observationally, individuals dealing with religious, mystic and meditative disciplines have a general state of calmness. In another study conducted, it has been put forward that meditation and yoga were associated with smaller amygdala [7].

Generally, studies conducted on patient groups have given different results. The common characteristic of these patient groups is having a chronic stress state. Exposition to this long-term stress, mostly since childhood, causes damages in limbic system structures such as amygdala [34] and hippocampus which have glucocorticoid receptor on them. However, our study was conducted on completely healthy young adults and the purpose of the study was to compare the cortisol level released against sudden stress and amygdala size.

Volumetric measurements have gained importance through imaging techniques. Symmetry or asymmetry of neuroanatomical structures distributed to both hemispheres is used as a prediction tool for clinicians in pathological processes [3]. An asymmetry is mentioned in the literature between right and left amygdala in terms of both function and size. In a meta-analysis [23] which examined 82 studies conducted on healthy individuals between 1990 and 2002 showed that the right amygdala size was bigger than that of the left amygdala. In our study, we found the right amygdala size statistically significantly bigger than that of the left hemisphere.

State-TraitAnxiety Inventory is one of the most used scales to assess anxiety. Anxiety has two components: state anxiety and trait anxiety [4]. In contrast trait anxiety, state anxiety shows psychological and 
physiological transient reactions directly associated with adverse situations at the given time [14]. So, we used state part of STAI to see anxiety scores of volunteers before exam. Occasional anxiety is part of life. People can feel anxious when faced with problems in work/school, before tests, before taking important decisions. Our volunteers got high anxiety scores before exam. Our findings are in line with the studies about exam stress $[24,28]$.

\section{Conflict of interest: None declared}

\section{REFERENCES}

1. Baur V, Hänggi J, Jäncke L. Volumetric associations between uncinate fasciculus, amygdala, and trait anxiety. BMC Neurosci. 2012; 13: 4, doi: 10.1186/1471-2202-13-4, indexed in Pubmed: 22217209.

2. Beheshtifar M, Hoseinifar H, Moghadam M. Effect procrastination on work-related stress. Eur J Econ Finance Admin Sci. 2011; 38: 59-64.

3. Duboc V, Dufourcq $P$, Blader $P$, et al. Asymmetry of the brain: development and implications. Annu Rev Genet. 2015; 49: 647-672, doi: 10.1146/annurev-genet-112414-055322, indexed in Pubmed: 26442849.

4. Fernández-Blázquez MA, Ávila-Villanueva M, López-Pina $J A$, et al. Psychometric properties of a new short version of the State-Trait Anxiety Inventory (STAI) for the assessment of anxiety in the elderly. Neurologia. 2015; 30(6): 352-358, doi: 10.1016/j.nrl.2013.12.015, indexed in Pubmed: 24484757.

5. Gatti R, Antonelli G, Prearo M, et al. Cortisol assays and diagnostic laboratory procedures in human biological fluids. Clin Biochem. 2009; 42(12): 1205-1217, doi: 10.1016/j. clinbiochem.2009.04.011, indexed in Pubmed: 19414006.

6. Gerritsen L, Rijpkema M, van Oostrom I, et al. Amygdala to hippocampal volume ratio is associated with negative memory bias in healthy subjects. Psychol Med. 2012; 42(2): 335-343, doi: 10.1017/S003329171100122X, indexed in Pubmed: 21740626.

7. Gotink RA, Vernooij MW, Ikram MA, et al. Meditation and yoga practice are associated with smaller right amygdala volume: the Rotterdam study. Brain Imaging Behav. 2018; 12(6): 1631-1639, doi: 10.1007/s11682-018-9826-z, indexed in Pubmed: 29417491.

8. Granger DA, Kivlighan KT, el-Sheikh M, et al. Salivary alpha-amylase in biobehavioral research: recent developments and applications. Ann N Y Acad Sci. 2007; 1098: 122-144, doi: 10.1196/annals.1384.008, indexed in Pubmed: 17332070.

9. Holmes AJ, Lee PH, Hollinshead MO, et al. Individual differences in amygdala-medial prefrontal anatomy link negative affect, impaired social functioning, and polygenic depression risk. J Neurosci. 2012; 32(50): 18087-18100, doi: 10.1523/JNEUROSCI.2531-12.2012, indexed in Pubmed: 23238724

10. Kim EJ, Pellman B, Kim JJ. Stress effects on the hippocampus: a critical review. Learn Mem. 2015; 22(9): 411-416, doi: $10.1101 / \mathrm{Im} .037291 .114$, indexed in Pubmed: 26286651 .
11. Koob GF. The role of CRF and CRF-related peptides in the dark side of addiction. Brain Res. 2010; 1314: 3-14, doi: 10.1016/j.brainres.2009.11.008, indexed in Pubmed: 19912996.

12. Kronenberg G, Tebartz van Elst L, Regen F, et al. Reduced amygdala volume in newly admitted psychiatric in-patients with unipolar major depression. J Psychiatr Res. 2009; 43(13): 1112-1117, doi: 10.1016/j.jpsychires.2009.03.007, indexed in Pubmed: 19394960.

13. Kudielka B, Hellhammer DH, Wüst S. Why do we respond so differently? Reviewing determinants of human salivary cortisol responses to challenge. Psychoneuroendocrinology. 2009; 34(1): 2-18, doi: 10.1016/j.psyneuen.2008.10.004.

14. Leal PC, Goes TC, da Silva LC, et al. Trait vs. state anxiety in different threatening situations. Trends Psychiatry Psychother. 2017; 39(3): 147-157, doi: 10.1590/2237-60892016-0044, indexed in Pubmed: 28813110.

15. Lederbogen F, Kirsch $P$, Haddad L, et al. City living and urban upbringing affect neural social stress processing in humans. Nature. 2011; 474(7352): 498-501, doi: 10.1038/ nature10190, indexed in Pubmed: 21697947.

16. LeDoux J. The emotional brain, fear, and the amygdala. Cell Mol Neurobiol. 2003; 23(4-5): 727-738, doi: 10.1023/a:1025048802629, indexed in Pubmed: 14514027.

17. Lee DoY, Kim E, Choi MHo. Technical and clinical aspects of cortisol as a biochemical marker of chronic stress. BMB Rep. 2015; 48(4): 209-216, doi: 10.5483/ bmbrep.2015.48.4.275, indexed in Pubmed: 25560699.

18. Lee SC, Amir A, Haufler D, et al. Differential recruitment of competing valence-related amygdala networks during anxiety. Neuron. 2017; 96(1): 81-88.e5, doi: 10.1016/j. neuron.2017.09.002, indexed in Pubmed: 28957678.

19. Malykhin N, Travis S, Sereshki A, et al. 132. Effects of cortisol on hippocampal subfields and centromedial amygdala volumes in healthy subjects and patients with major depressive disorder. Biol Psychiatry. 2018; 83(9): S54, doi: 10.1016/j.biopsych.2018.02.150.

20. Marin MF, Lord C, Andrews J, et al. Chronic stress, cognitive functioning and mental health. Neurobiol Learn Mem. 2011; 96(4): 583-595, doi: 10.1016/j.nlm.2011.02.016, indexed in Pubmed: 21376129.

21. Morris JS, Ohman A, Dolan RJ. A subcortical pathway to the right amygdala mediating "unseen" fear. Proc Natl Acad Sci U S A. 1999; 96(4): 1680-1685, doi: 10.1073/ pnas.96.4.1680, indexed in Pubmed: 9990084

22. Özel $Y$, Karabulut AB. Daily living and stress management. Turkish J Health Sci Res. 2018; 1(1): 48-56.

23. Pedraza O, Bowers D, Gilmore R. Asymmetry of the hippocampus and amygdala in MRI volumetric measurements of normal adults. J Int Neuropsychol Soc. 2004; 10(5): 664-678, doi: 10.1017/\$1355617704105080, indexed in Pubmed: 15327714.

24. Ping LT, Subramaniam K, Krishnaswamy S. Test anxiety: state, trait and relationship with exam satisfaction. Malays J Med Sci. 2008; 15(2): 18-23, indexed in Pubmed: 22589620.

25. Schuhmacher A, Mössner R, Jessen F, et al. Association of amygdala volumes with cortisol secretion in unipolar depressed patients. Psychiatry Res. 2012; 202(2): 96-103, 
doi: 10.1016/j.pscychresns.2011.09.007, indexed in Pubmed: 22698761

26. Sharma D. Physiology of stress and its management. J Med Study Res. 2018; 1(1): 1-5, doi: 10.24966/msr$5657 / 100001$.

27. Sharma M, Rush SE. Mindfulness-based stress reduction as a stress management intervention for healthy individuals: a systematic review. J Evid Based Complementary Altern Med. 2014; 19(4): 271-286, doi: 10.1177/2156587214543143, indexed in Pubmed: 25053754.

28. Spangler G. Psychological and physiological responses during an exam and their relation to personality characteristics. Psychoneuroendocrinology. 1997; 22(6): 423-441, doi: 10.1016/s0306-4530(97)00040-1.

29. Spiga F, Walker JJ, Terry JR, et al. HPA axis-rhythms. Compr Physiol. 2014; 4(3): 1273-1298, doi: 10.1002/cphy. c140003, indexed in Pubmed: 24944037.

30. Tottenham N, Hare TA, Quinn BT, et al. Prolonged institutional rearing is associated with atypically large amygdala volume and difficulties in emotion regula- tion. Dev Sci. 2010; 13(1): 46-61, doi: 10.1111/j.14677687.2009.00852.x, indexed in Pubmed: 20121862.

31. Tottenham N, Sheridan MA. A review of adversity, the amygdala and the hippocampus: a consideration of developmental timing. Front Hum Neurosci. 2009; 3: 68-77, doi: 10.3389/ neuro.09.068.2009, indexed in Pubmed: 20161700.

32. Tufan AE, Yaluğ i. Love phenomenon and neurobiology of love relations. Curr App Psychiatry. 2010; 2(4): 443-456.

33. van der Plas EAA, Boes AD, Wemmie JA, et al. Amygdala volume correlates positively with fearfulness in normal healthy girls. Soc Cogn Affect Neurosci. 2010; 5(4): 424-431, doi: 10.1093/scan/nsq009, indexed in Pubmed: 20150341.

34. Wang Q, Verweij EWE, Krugers HJ, et al. Distribution of the glucocorticoid receptor in the human amygdala; changes in mood disorder patients. Brain Struct Funct. 2014; 219(5): 1615-1626, doi: 10.1007/s00429-013-0589-4, indexed in Pubmed: 23748930.

35. Yıldız M, Güç K, Erdem S. Examine attitudes to cope with stress in terms of human values: a study on public employees. Sci J Turkish Military Academy. 2015; 25(1): 41-61. 